\title{
The Use of Epidural Anaesthesia over General Anaesthesia in Ruminants
}

\author{
Olaifa $\mathrm{AK}^{* 1}$ and Oluranti $\mathrm{OI}^{2}$ \\ ${ }^{1}$ Department of Veterinary Surgery and Radiology, University of Ibadan, Nigeria \\ ${ }^{2}$ Department of Physiology, University of Ibadan, Nigeria
}

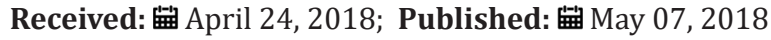

*Corresponding author: Olaifa AK, Department of Veterinary Surgery and Radiology, University of Ibadan, Nigeria

\begin{abstract}
General anaesthesia is an essential component of modern medicine. It is a drug induced reversible condition that includes specific behavioral and physiological traits - unconsciousness, amnesia, analgesia, and akinesia- with concomitant stability of the autonomic, cardiovascular, respiratory, and thermoregulatory systems. General anesthesia in ruminants has inherent risks such as regurgitation of ruminal contents, excessive salivation and the possibility of pulmonary aspiration; therefore it is not always recommended and local or regional techniques may be used instead. Epidural anaesthesia is a central neuraxial block technique which is used regularly in veterinary practice for treatment of different obstetrical and surgical interferences in the perineal, sacral, lumbar, and caudal parts of the thoracic region of domestic animals. There have been several scientific researches on the use of epidural anesthesia in ruminants and other species for various surgical and laboratory procedures. In conclusion, the disadvantages of general anaesthesia outweigh the advantages especially in ruminant specie. Therefore, epidural anaesthesia is the most preferred.
\end{abstract}

Keywords: Anaesthesia; Ruminant; Epidural; General

\section{Introduction}

Anaesthesia is an indispensible prerequisite for most surgical interventions both in humans and in animals Muhammad [1] It is supposed to provide reversible unconsciousness, amnesia/ analgesia, muscle relaxation, and immobility with minimal adverse effects, rapid and smooth recovery of protective reflex and psychomotor function Bajwa [2] Hemming. General anaesthesia is the controlled and reversible loss of consciousness for many surgical procedures that warrant effective control of pain and movement.

General anaesthesia is an essential component of modern medicine. It is a drug induced, reversible condition that includes specific behavioral and physiological traits - unconsciousness, amnesia, analgesia, and akinesia- with concomitant stability of the autonomic, cardiovascular, respiratory, and thermoregulatory systems (Brown [3]. In the practice of medicine (especially surgery and dentistry), anaesthesia is a state of temporary induced loss of sensation or awareness. It may include analgesia (relief from or prevention of pain), paralysis (muscle relaxation), amnesia (loss of memory), or unconsciousness A patient under the effects of anesthetic drugs is referred to as being anesthetized. Anesthesia enables the painless performance of medical procedures that would cause severe or intolerable pain to an unanesthetized patient. Three broad categories of anaesthesia exist: General anesthesia suppresses central nervous system activity and results in unconsciousness, total lack of sensation and loss of protective reflexes. Sedation suppresses the central nervous system to a lesser degree, inhibiting both anxiety and creation of long-term memories without resulting in unconsciousness.

Regional anesthesia and local anesthesia, which block transmission of nerve impulses between a targeted part of the body and the central nervous system, causing loss of sensation in the targeted body part. A patient under regional or local anesthesia remains conscious, unless general anaesthesia or sedation is administered at the same time. Two broad classes exist: 
a) Peripheral blockade inhibits sensory perception in an isolated part of the body, such as numbing a tooth for dental work or administering a nerve block to inhibit sensation in an entire limb.

b) Central, or neuraxial, blockade administers the anesthetic in the region of the central nervous system itself, suppressing incoming sensation from outside the area of the block. Examples include epidural anaesthesia and spinal anaesthesia.

To achieve the goals of anesthesia, drugs act on different but interconnected parts of the nervous system. Hypnosis, for instance, is generated through actions on the nuclei in the brain and is similar to the activation of sleep. The effect is to make the patient less aware and less reactive to noxious stimuli Miller and Ronald [4].

Loss of memory (amnesia) is created by action of drugs on multiple (but specific) regions of the brain. Memories are created as either declarative or non-declarative memories in several stages (short-term, long-term, and long-lasting) the strength of which is determined by the strength of connections between neurons termed synaptic plasticity. Each anesthetic produces amnesia through unique effects on memory formation at variable doses. Inhalational anesthetics will reliably produce amnesia through general suppression of the nuclei at doses below those required for loss of consciousness. Drugs like midazolam produce amnesia through different pathways by blocking the formation of long-term memories.

Tied closely to the concepts of amnesia and hypnosis is the concept of consciousness. Consciousness is the higher order process that synthesizes information. For instance, the "sun" conjures up feelings, memories and a sensation of warmth rather than a description of a round, orange warm ball seen in the sky for part of a 24 hour cycle. Likewise, a person can have dreams (a state of subjective consciousness) during anesthetic or have consciousness of the procedure despite having no indication of it under anesthetic. It is estimated that $22 \%$ of people dream during general anesthesia and 1 or 2 cases per 1000 have some consciousness termed "awareness during general anesthesia” Miller and Ronald [5].

\section{Advantages and Disadvantages in Ruminants}

General anesthesia in ruminants has inherent risks such as regurgitation of ruminal contents, excessive salivation and the possibility of pulmonary aspiration; therefore it is not always recommended and local or regional techniques may be used instead (Hall and Clarke [5]) Sheep and goats are ideally suited to local analgesic techniques under manual restraint with or without sedation (Taylor [6]).

Local anesthesia is any technique to induce the absence of sensation in a specific part of the body, generally for the aim of inducing local analgesia, that is, local insensitivity to pain, although other local senses may be affected as well. It allows patients to undergo surgical and dental procedures with reduced pain and distress. In many situations, such as cesarean section, it is safer and therefore superior to general anesthesia. It is also used for relief of non-surgical pain and to enable diagnosis of the cause of some chronic pain conditions. Anesthetists sometimes combine both general and local anesthesia techniques.

However, there are other important reasons for using epidurals, including a reduction in the incidence of perioperative pulmonary and cardiac complications in certain settings Ballantyne [7] Beattie [8]. The evidence that epidurals offer better postoperative analgesia than systemic opioids is unequivocal, Block [9] and the risk of major complications from epidurals inserted in the perioperative period is low Cook [10].

Regional anaesthesia certainly provides high quality pain relief Popping [11]. However, it may carry a risk of neurological deficit. A systematic review of neurological complications after regional anaesthesia cited incidences of neuropathy of 1.48:100 for axillary block and of 2.84:100 for interscalene block, Brull [12] but permanent neurological injury is very rare. The balance of risks and benefits has to be considered in the context of the particular patient, operation, and regional block.

\section{Epidural Anaesthesia}

Epidural anaesthesia is a central neuraxial block technique which is used regularly in veterinary practice for treatment of different obstetrical and surgical interferences in the perineal, sacral, lumbar, and caudal parts of the thoracic region of domestic animals. Epidural anesthesia using local anesthetic drugs has been a common technique used in veterinary medicine to perform surgical procedures since the 1950s in North America and Europe. With the advent of safer injectable and inhalational anesthetic drugs and accessibility to anesthetic equipment, the use of epidural anesthesia became less frequent in the following years. In the late 1980s, however, with the recognition of opioids' analgesic actions on the spinal cord, the use of epidural analgesia became an important tool that has re-emerged in intra- and postoperative epidural techniques to provide analgesia and anesthesia in veterinary medicine Valverde [13]. Epidural administration (from Ancient Greek ع́ $\pi i$, "on, upon" + dura mater) is a medical route of administration in which a drug or contrast agent is injected into the epidural space of the spinal cord. Techniques such as epidural analgesia and epidural anaesthesia employ this route of administration. The injection can result in a loss of sensation, including the sensation of pain by blocking the transmission of signals through nerve fibers in or near the spinal cord. Injecting medication into the epidural space is primarily performed for analgesia. This may be performed using a number of different techniques and for a variety of reasons. Additionally, some of the side-effects of epidural analgesia may be beneficial in some circumstances (e.g., vasodilation may be beneficial if the subject has peripheral vascular disease). When a catheter is placed into the epidural space a continuous infusion can be maintained for several days, if needed. Epidural analgesia may be used: 
a) For analgesia alone, where surgery is not contemplated. An epidural injection or infusion for pain relief (e.g. in childbirth) is less likely to cause loss of muscle power, but has to be augmented to be sufficient for surgery.

b) As an adjunct to general anaesthesia. The anaesthetist may use epidural analgesia in addition to general anaesthesia. This may reduce the patient's requirement for opioid analgesics. This is suitable for a wide variety of surgery, for example gynaecological surgery (e.g. hysterectomy), orthopaedic surgery (e.g. hip replacement), general surgery (e.g. laparotomy) and vascular surgery (e.g. open aortic aneurysm repair).

c) As a sole technique for surgical anaesthesia. Some operations, most frequently Caesarean section, may be performed using an epidural anaesthetic as the sole technique. This can allow the patient to remain awake during the operation. The dose required for anaesthesia is much higher than that required for analgesia.

d) For post-operative analgesia, after an operation where the epidural technique is employed as the sole anaesthetic, or in conjunction with general anaesthesia. Analgesics are administered into the epidural space typically for a few days after surgery, provided a catheter has been inserted. Through the use of a patient-controlled epidural analgesia (PCEA) infusion pump, a person can supplement an epidural infusion with occasional doses of pain medication through an epidural catheter.

e) For the treatment of back pain. Injection of analgesics and steroids into the epidural space may improve some forms of back pain.

f) For the treatment of chronic pain or palliation of symptoms in terminal care, usually in the short- or mediumterm.

The epidural space is more difficult and risky to access as one ascends the spine (because the spinal cord gains more nerves as it ascends and fills the epidural space leaving less room for error), so epidural techniques are most suitable for analgesia anywhere in the lower body and as high as the chest. They are (usually) much less suitable for analgesia for the neck, or arms and are not possible for the head (since sensory innervation for the head arises directly from the brain via cranial nerves rather than from the spinal cord via the epidural space).

The epidural space is the space inside the bony spinal canal but just outside the dura mater ("dura"). In contact with the inner surface of the dura is another membrane called the arachnoid mater ("arachnoid"). The cerebrospinal fluid that surrounds the spinal cord is contained by the arachnoid mater. In adults, the spinal cord terminates around the level of the disc between L1 and L2 (in neonates it extends to L3 but can reach as low as L4), below which lies a bundle of nerves known as the cauda equina ("horse's tail"). Hence, lumbar epidural injections carry a low risk of injuring the spinal cord. Insertion of an epidural needle involves threading a needle between the bones, through the ligaments and into the epidural potential space taking great care to avoid puncturing the layer immediately below containing CSF under pressure.

There have been several scientific researches on the use of epidural anesthesia in ruminants and other species for various surgical and laboratory procedures. Olaifa [14] reported the physiological and biochemical effects of epidural lidocaine with adrenaline in pregnant West African dwarf goats and this will serve as reference point for surgeons when operating on pregnant animals under epidural anaesthesia especially during caesarean section and rectal tear repairs. Olaifa [15] used epidural anaesthesia with plain lignocaine in naturally PPR infected WAD goats to produce effective anaesthesia which can be used for surgical procedures in such animals. Kisani and his colleagues [16] compared the effects of duration of epidural anaethesia in West African dwarf goats using ketamine $\mathrm{Hcl}$, lidocaine $\mathrm{Hcl}$ and xylazine $\mathrm{Hcl}$; lidocaine-distilled water and lidocaine-magnesium sulfate mixture Sadegh 17] Also, comparing the efficacy of medetomidine $\mathrm{HCl}$ and lignocaine $\mathrm{HCl}$ as epidural anesthetic in Buffalo Calves Akbar [18] Epidural morphine improves analgesia in goats undergoing hindlimb3 and abdominal2 surgery Pablo [19]; Hendrickson et al. [20]. Lumbosacral epidural lidocaine-epinephrine, lidocaine-xylazine and bupivacaine provide prolonged anaesthesia that may contribute to pain relief in the immediate postoperative period in animals undergoing surgical procedures involving the flank, perineum and hindlimb Rostami and Vesal [21]. The combination of bupivacaine $(0.25 \mathrm{mg} \mathrm{kg}-1)$ plus ketamine (1.25mg kg-1) has been used safely for epidural analgesia in sheep without any marked side effects Dadafarid and Najafpour [22]. Epidural administration of tramadol alone provided good anti-nociception in the perineal region, with a prolonged duration of effect with no ataxia, however, tramadol-lidocaine combination resulted in a rapid onset time and prolonged duration which may be useful in clinical practice for a single-dose epidural administration to enable surgical and obstetrical procedures of long duration to be completed Dehkordi. Epidural injection of lignocaine produced a longer duration of antinociception with lower frequency of pain associated behavioural changes in goat subjected to castration with the use of high tension band Ajadi [23]. Thoracolumbar epidural technique also promotes satisfactory analgesia in the animal's flank, and a larger anaesthetized area. The combination of $0.5 \%$ bupivacaine and methadone was shown to be more effective in promoting analgesia, with a short-lasting ataxic period and no undesirable effects when compared to bupivacaine alone Silva $[24,25]$.

\section{Conclusion}

In conclusion, from the scientific literatures and information gathered, the disadvantages of general anaesthesia outweigh the advantages especially in ruminant specie. Therefore, epidural anaesthesia is the most preferred. 


\section{References}

1. Muhammad N, Zafar MA, Muhammad G, Masood MZ, Manzoor A, et al. (2009) "Comparative anaesthetic efficacy of propofol, thiopental sodium and combination of propofol with ketamine hydrochloride in dogs," Pakistan Veterinary Journal 29(1): 11-15.

2. Bajwa SJ, SK Bajwa, J Kaur (2010) “Comparison of two drugs combination in total intravenous anaesthetics: propofol-ketamine and propotolFentanyl," Saudi Journal of Anaesthesia 4(2): 72-79.

3. Brown EN, Lydic R, Schiff ND (2010) General Anesthesia, Sleep, and Coma. The New England Journal of Medicine 363(27): 2638-2650

4. Miller, Ronald D, Erikson Lars I, Fleisher Lee A, Wiener-Kronish Jeanine P, et al. (Eds.), (2010) Miller's Anesthesia $7^{\text {th }}$ Edn. Elsevier, USA: Churchill Livingstone.

5. Hall LW, Clarke KW (1991) Veterinary Anaesthesia ( $9^{\text {th }}$ edn.), London: Baillière Tindall 236: 186-187.

6. Taylor PM (1991) Anesthesia in sheep and goats. In Pract 13: 31-36.

7. Ballantyne JC, Carr DB, De Ferranti S, Suarez T, Lau J, et al. (1998) The comparative effects of postoperative analgesic therapies on pulmonary outcome: cumulative meta-analyses of randomized, controlled trials. Anesth Analg 86(3): 598-612.

8. Beattie WS, Badner NH, Choi PT (2003) Meta-analysis demonstrates statistically significant reduction in postoperative myocardial infarction with the use of thoracic epidural analgesia. Anesth Analg 97(3): 919920.

9. Block BM, Liu SS, Rowlingson AJ, Cowan AR, Cowan JA, et al. (2003) Efficacy of postoperative epidural analgesia: a meta-analysis. J Am Med Assoc 290(18): 2455-2463.

10. Cook TM, Counsell D, Wildsmith JA (2009) Major complications of central neuraxial block: report on the Third National Audit Project of the Royal College of Anaesthetists. Br J Anaesth 102(2): 179-190.

11. Popping DM, Zahn PK, Van Aken HK, Dasch B, Boche R, et al. (2008) Effectiveness and safety of postoperative pain management: a survey of 18925 consecutive patients between 1998 and 2006 (2nd revision): a database analysis of prospectively raised data. Br J Anaesth 101(6): 832-840.

12. Brull R, McCartney CJ, Chan VW, El-Beheiry H (2007) Neurologica complications after regional anesthesia: contemporary estimates of risk. Anesth Analg 104(4): 965-974.

13. Valverde A (2008) Epidural Analgesia and Anesthesia in Dogs and Cats Vet Clin Small Anim 38 (6): 1205-1230.
14. Olaifa Abayomi Kayode (2017) Studies on Epidural anaesthesia using lidocaine with Adrenaline on Hemato-biochemical responses in pregnant West African Dwarf goats. E3 Journal of Medical Research 6(2): 012-015.

15. Olaifa AK, Oguntoye CO, Brown A (2017) Anaesthetic Indices and Vital Parameters of PPR-Infected West African Dwarf Goats after Epidural Lignocaine Anaesthesia. Afr. J. Biomed. Res 20: 59- 63.

16. Kisani AI, Faeren PK and Elsa AT (2017) Comparative effects of duration of epidural anaesthesia in West African Dwarf goats using Ketamine HCL, Lidocaine HCL and Xylazine HCL. Inter J Vet Sci 6(1): 41-44.

17. Saied H Dehkordi, Amin Bigham-Sadegh, Razieh Geram (2012) Evaluation of anti-nociceptive effect of epidural tramadol, tramadollidocaine and lidocaine in goats. Veterinary Anaesthesia and Analgesia 39(1): 106-110.

18. Akbar H, MA Khan, SG Bokhari, Ahmad, HM Khan, et al. (2014) Comparative efficacy of medetomidine $\mathrm{HCl}$ and lignocaine $\mathrm{HCl}$ as epidural anesthetic in buffalo calves. Pak Vet J 34(3): 377-380.

19. Pablo LS (1993) Epidural morphine in goats after hindlimb orthopedic surgery. Vet Surg 22(4): 307-310.

20. Hendrickson DA, Kruse-Elliott KT, Broad stone RV (1996) A comparison of epidural saline, morphine, and bupivacaine for pain relief after abdominal surgery. Vet Surg 25: 83-87.

21. Rostami M, Vesal N (2012) The effects of adding epinephrine or xylazine to lidocaine solution for lumbosacral epidural analgesia in fat-tailed sheep. J S Afr Vet Assoc 83(1)

22. Hadi Dadafarid H, Najafpour A (2008) Epidural Analgesia with Bupivacaine, Ketamine, and the Combination of Bupivacaine and Ketamine in Sheep. Iranian Journal of Veterinary Surgery 3(1): 19-28.

23. Ajadi RA, AO Owanikin, MM Martins, OS Gazal (2012) Effect of epidural tramadol and lignocaine onphysiological and behavioural changes in goats subjected to castration with a high tension band, New Zealand Veterinary Journal 60(6): 344-348.

24. Priscila dos Santos Silva, Paulo Fantinato-Neto, André Nicolai Elias Silva, Eduardo Harry Birgel, Adriano Bowfin Carregaro (2017) Thoracolumbar epidural anaesthesia with $0.5 \%$ bupivacaine with or without methadone in goats Irish Veterinary Journal 70(15): 1-7.

25. Sadegh A, BZ. Shafiei, SD Nazhvani (2009) Comparison of epidural anesthesia with lidocaine-distilled water and lidocaine-magnesium sulfate mixture in goat. Vet arhiv 79(1): 11-17.

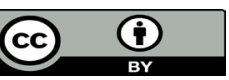

This work is licensed under Creative Commons Attribution 4.0 License

Submission Link:

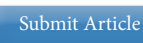

DOI: $10.32474 /$ CDVS.2018.01.000111

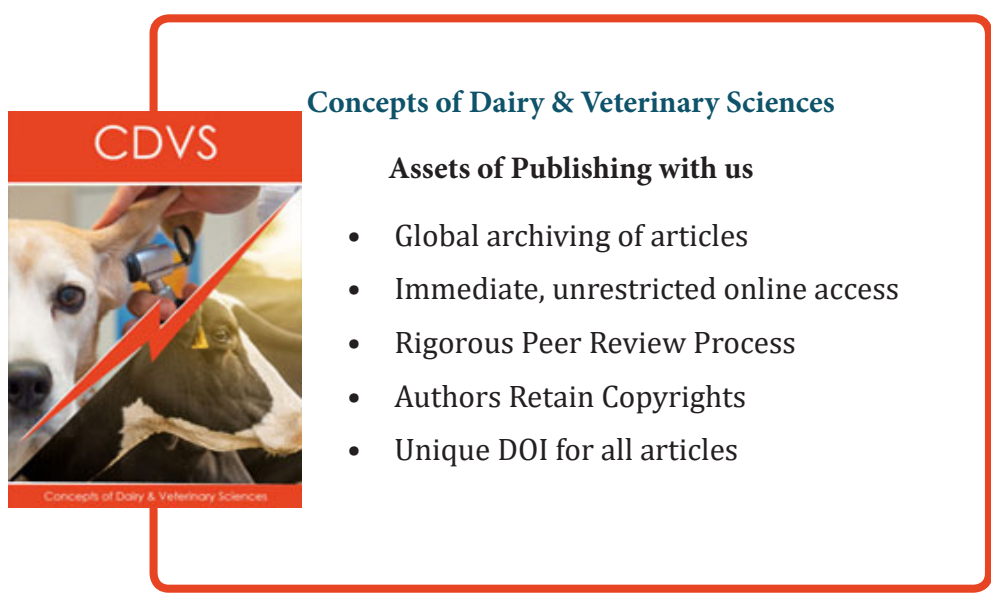

